\title{
Il digitale e la rappresentazione: la seconda linea e il castello dimenticato di Fiumedinisi
}

Digital and representation: the second line and the forgotten castle of Fiumedinisi

\section{Giuseppe Di Gregorio}

Dipartimento di Ingegneria e Architettura - Università degli Studi di Catania, Catania, Italy

giuseppe.digregorio@dau.unict.it / giuseppe.digregorio@unict.it

\begin{abstract}
The Belvedere Castle of Fiumedinisi (ME) belongs to that historical heritage of Sicily characterized by abandoned and forgotten military architecture. Along the Ionian coast the defensive problem has been particularly felt over time, due to the proximity of the Turkish coast, the Middle East and the African one. The first defensive line was the coastal one, defined by principals placed on the sea in a strategic position for direct control of the coast. They were part of this group: the Maniace castle of Syracuse, that of Augusta, of Brucoli, of Catania, of Acicastello, the Tocco of Acireale, Schisò in the territory of Giardini, Capo Sant'Alessio, the Saracen Tower of Roccalumera, Capo Grosso in Ali , San Salvatore in Messina. Along the eastern side of the Peloritani mountains from Calatabiano to Messina, the island's defensive strategy also included a second line of fortifications, which controlled a more distant horizon from their position. These include the castle of Calatabiano, Taormina, Castelmola, Forza d'Agrò, Savoca, Fiumedinisi, Scaletta Zanclea, Santo Stefano di Briga, Matagrifone. Among them, the Belvedere castle of Fiumedinisi, at a critical distance from the village, so as to be in a state of neglect, among those listed is that which is in the worst conditions. In stark contrast to the dignity and history of the site and territory of Fiumedinisi, dating back to the Greek period. In this work we propose the survey of the castle with digital, photogrammetric technologies, Structure From Motion (SFM) and dense matching, to arrive at a $3 \mathrm{D}$ documentation and graphic drawings, considering that to date there are no significant scientific surveys and representations of this abandoned fortress.
\end{abstract}

Keywords: Relief, 3D representation, digital survey, SFM, photogrammetry.

\section{Introduzione}

Con riferimento al loro stato di conservazione $\mathrm{i}$ castelli si suddividono in due tipologie ben distinte. La prima comprende tutte le fortificazioni che si trovano attualmente all'interno del perimetro urbano, queste sono essenzialmente in buono stato di conservazione, mentre la seconda è riferita a quelle fortificazioni che sono al di fuori del perimetro, a volte anche a notevole distanza, per queste è innegabile il loro abbandono e il loro cattivo stato di conservazione. Di alcune fortezze non vi è più traccia, la loro passata esistenza è documentata nelle fonti di archivio. Di quelle ancora visibili, nel migliore dei casi oggi rimangono dei ruderi, talvolta leggibili, a volte no. Questa categoria rappresenta la fascia più debole sul territorio, di cui talvolta non esistono lavori sistematici di rilievo che ne documentino la consistenza, come nel caso in esame. 


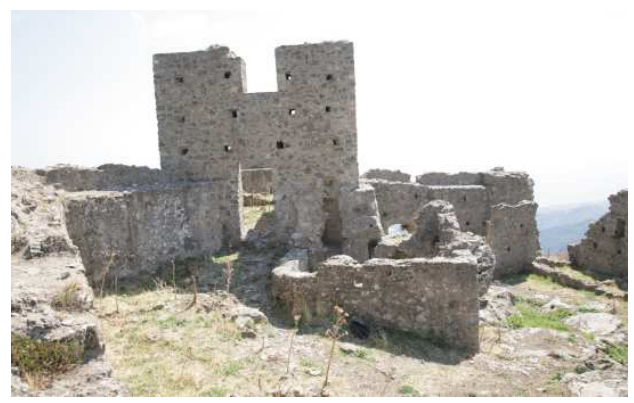

Fig. 1. Il castello di Fiumedinisi, scorcio dell'interno.

La fortificazione fa parte di quella seconda linea difensiva della costa ionica che sfrutta l'orografia dei monti Peloritani, che si inerpicano rapidamente a partire dal litorale ionico. Così il castello consentiva a poca distanza dalla linea della costa di controllare il territorio sottostante sino al mare. Ma il vantaggio era quello di avere una linea d'orizzonte più ampia e più distante da controllare. Gli altri castelli che appartengono a questa seconda linea difensiva sono quello di Taormina, Castelmola, Forza d'Agrò, Savoca, Scaletta, Messina. Inoltre la posizione del Castello Belvedere consentiva la comunicazione visiva con il Castello di Sant'Alessio, di Forza d'Agrò e di Scaletta Zanclea. Dopo la riconquista dei Normanni, l'importanza strategica dell'isola nel Mediterraneo è ancora confermata anche dopo la caduta di Costantinopoli (1453), ma anche nel secolo successivo in un mare infestato da pirati e nemici. L'azione di Carlo $V$ darà avvio ad operazioni di architettura militare mirate a dotare l'isola di torri, mura e baluardi, il pericolo al suo tempo era rappresentato dalle invasioni dei turchi e l'isola per la sua posizione di centralità nel mediterraneo sentiva particolarmente questa pressione. Si comprende quindi l'importanza dei baluardi difensivi in ogni tempo nell'isola e soprattutto nella costa ionica.

\section{Cenni storici}

Di seguito si danno alcuni cenni storici al solo scopo di mostrare il significato e l'importanza che ha avuto il castello nello scorso millennio. Gli storici locali ritengono che la fortezza di Nikos o forse Vicos, potrebbe essere stata realizzata dagli Arabi nel X secolo, in sostituzione di un preesistente tempio di fattura greca, edificato in onore del dio Dioniso e voluto dai nativi che abitavano il villaggio sottostante, denominato Nisa. Tra essi C. Gregorio (Gregorio, 2013, p. 222.) ritiene che: "La quasi omonimia tra Nikos e Nisa, la corrispondenza delle distanze riportate dall'Amari, le fondamenta pre-arabe del castello, situato in posizione dominante sulla valle e l'esistenza di una città già abitata proprio alle pendici dello stesso monte, il ritrovamento nel sito di re-

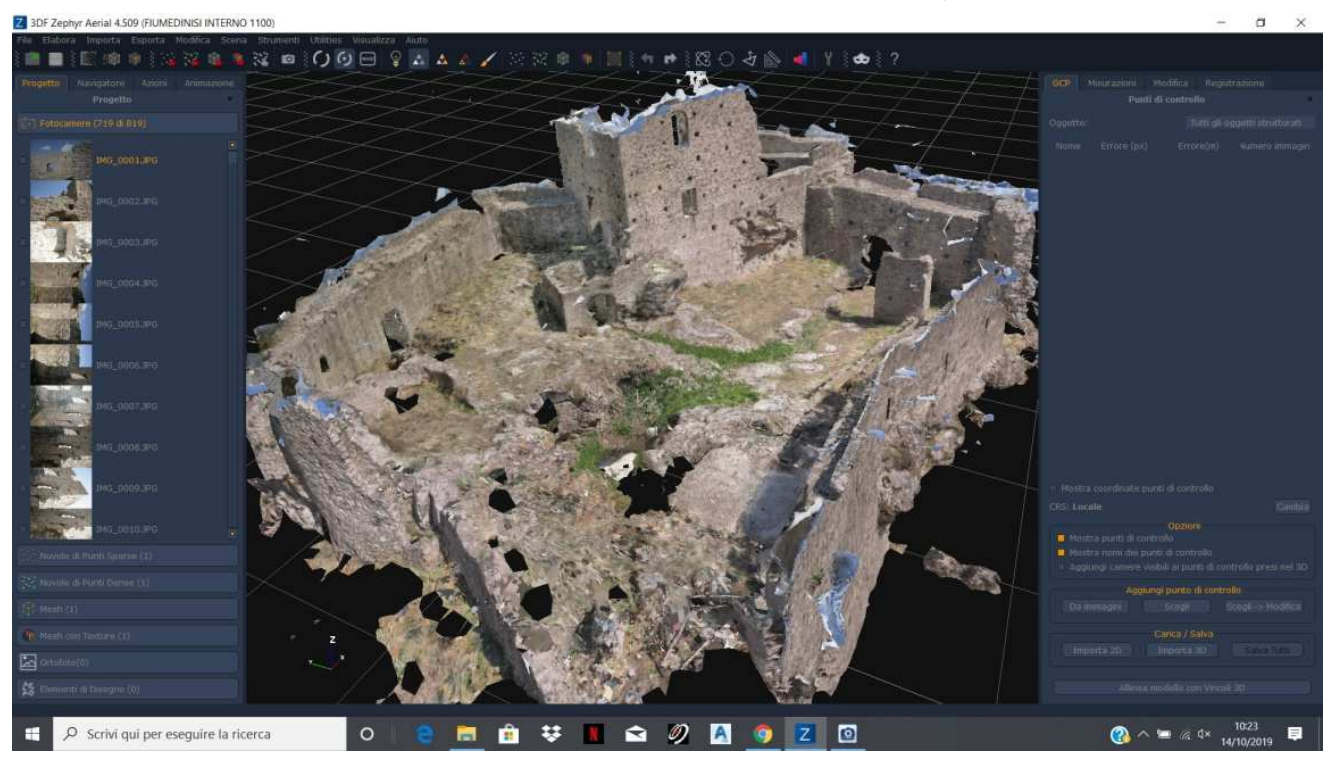

Fig. 2. Castello Belvedere, elaborazione 3D con mesh e texture. 
perti archeologici databili al IX-X secolo, sono tutti elementi molto rilevanti che confermano quanto sopra affermato" (Gregorio, 2013, p. 49). Altri studi hanno rinvenuto nel terrazzamento sottostante il castello l'area archeologica, riconducibile all'antica colonia greca di Nisa. Nel periodo successivo alla riconquista, i Normanni ampliarono il castello, apportando delle modifiche e adibendolo a residenza del feudatario del luogo. Variava però il rapporto con il nucleo abitato che migrava a valle, in prossimità del fiume. Le notizie certe arrivano dal 1197, anno in cui il castello ospitò Enrico VI con la famiglia e con il seguito. La venuta a Fiumedinisi dell'imperatore coincise con la sua scomparsa avvenuta lo stesso anno, quindi seguirono gli angioini nel 1266 con la figura di Enrico d'Angiò. Poi è un continuo avvicendarsi di nomi illustri che hanno fatto la storia dell'isola e dell'Italia meridionale, fino al 1392 anno in cui la fortezza fu concessa dal Re Martino a Tommaso Romano Colonna. Questa è l'ultima famiglia che lo ha detenuto, ma che nei fatti lo ha abbandonato sia per la precarietà della sua posizione, sia per le altre immense ricchezze possedute (Vallone, 2005.). Oltre al collegamento visivo con le altre fortificazioni di cui si è detto, il castello lo aveva con la torre di avvistamento e di difesa del sottostante centro abitato, oggi facente parte della chiesa di San Pietro, e anche con una torre dell'epoca ubicata nell'omonima contrada ${ }^{1}$. Sappiamo infine che nel 1871, il Duca di Cesarò discendente della famiglia Romano Colonna, concede per pascolo il castello e lo spiazzo adiacente. Successivamente la legge n. 1089 del 1939 permise di espropriare il castello da parte del comune e dichiararlo monumento nazionale. Tra il 2004 e il 2005 sono stati realizzati dei lavori di restauro che hanno riguardato il consolidamento delle parti strutturali, la cisterna e la strada di accesso.

\section{L'impianto architettonico}

Il nome attuale deriva dal toponimo del monte Belvedere, attestato ad una quota di circa $743 \mathrm{~m}$. La pianta è impostata su di un poligono irregolare, sulla sommità del crinale, pensato per non lasciare spazi indifesi. Infatti i lati a nord-est e a sud-ovest presentano un orografia impervia, quasi scoscesa sulle due vallate sottostanti. Nella sommità del perimetro murario trova spazio un camminamento di ronda. Dell'impianto originario sono ancora visibili i ruderi di alcuni muri perimetrali, consolidati nel secolo scorso da un opportuno intervento. L'intero perimetro murario presenta in parte una merlatura e delle feritoie riconducibili al periodo precedente

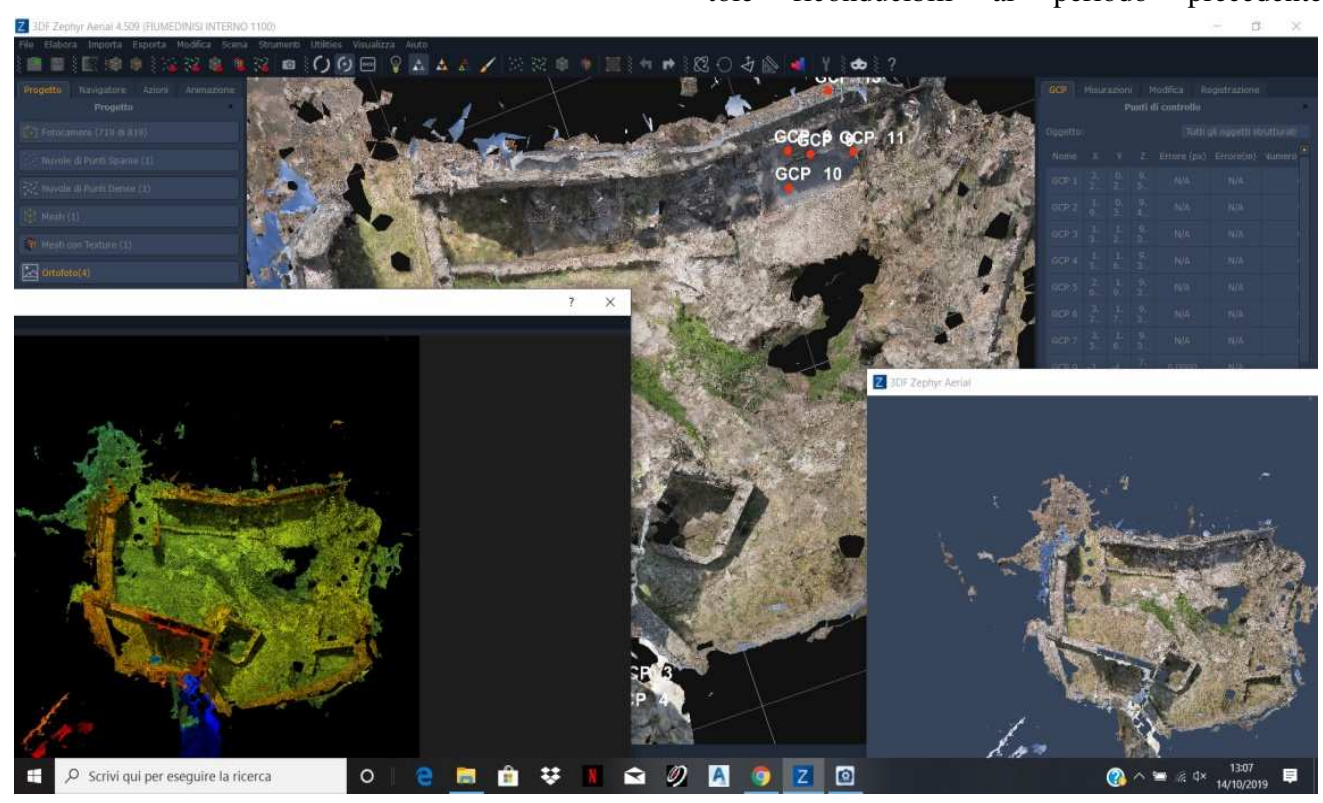

Fig. 3. Castello Belvedere, inserimento punti di controllo e generazione ortofoto. 
all'avvento delle armi da fuoco. All'interno diversi ruderi fanno intuire le probabili funzioni degli spazi e degli ambienti, tra essi è ancora oggi visibile la cisterna di dimensioni considerevoli con una profondità di circa $4 \mathrm{~m}$. Esternamente a tratti irregolari sono visibili altri ruderi riconducibili a un bastione murario. Va evidenziata la presenza di alcune grotte, in parte scavate nella roccia, di cui si può ipotizzare l'utilizzo come prigioni nel Medioevo e in precedenza come abitazioni rupestri. Diversi sono gli accessi al castello, ma quello ad oggi praticato è collocato nella zona retrostante, quella rivolta a nord, scandito da una scalinata lastricata in pietra di recentemente ripristinata.

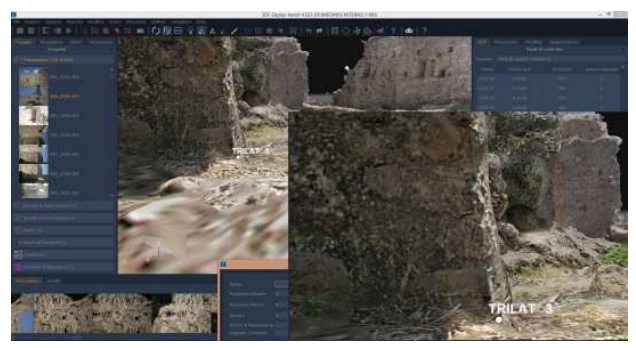

Fig. 4. Castello Belvedere, punto di controllo di una trilaterazione in prossimità di uno spigolo.

\section{La fase di rilievo}

Per la pianta si è utilizzato il metodo diretto di trilaterazione, tramite distanziometro elettronico Disto-D8 della Leica dotato di sensore di inclinazione, utilizzando la possibilità di rilevare misure su di un piano orizzontale, operazione assicurata dalla bolla torica presente sullo strumento. Per la graficizzazione delle parti degradate si è utilizzata l'ortofoto ottenuta dal modello 3D, scalato e referenziato con le misure provenienti dalle trilaterazioni eseguite con il distanziometro. Per le parti in elevazione l'occasione del rilievo è scaturita dalla possibilità di utilizzare le potenzialità, in continua e rapida evoluzione, della metodologia 3D SFM e dense matching a partire da un set fotografico eseguito tramite una camera reflex. Sebbene le applicazioni delle tecniche SFM per l'archeologia vengono utilizzate ormai da anni dagli archeologi e dai professionisti, la loro rapidissima evoluzione rende necessario verificare di continuo le nuove release, sia per i miglioramenti dei moduli già in uso sia per le parti innovative. Questi programmi si pongono nel settore della fotogrammetria, ma la loro possibilità di restituzione grafica ancor oggi è inferiore ai software di fotogrammetria classica di qualche decennio addietro. Il software utilizzato è lo Zephyr messo a disposizione dalla 3DFlow nella modalità aerial. Il programma, già utilizzato e testato nelle precedenti release in diversi casi studio per manufatti regolari, sia architettonici che archeologici, nel presente lavoro è stato sperimentato per i ruderi del castello: una situazione che permette di verificare accuratamente il software. L'utilizzo di un laser scanner avrebbe portato a risultati più gestibili in termini di precisione a fronte di costi molto onerosi, il vantaggio della metodologia SFM è quello di essere più speditiva e di avere dei costi molto bassi, di contro richiede continue verifiche nelle varie fasi. L'attenzione e la validazione è stata posta su quattro aspetti essenziali:

1) ottimizzazione delle fotografie

2) accurata selezione delle immagini

3) parametri di elaborazione SFM

4) restituzione grafica

La ripresa fotografica ha riguardato complessivamente un set di 819 fotografie eseguite con una camera full frame reflex EOS 5D, con ottica zoom 24-105 mm, che ben si è adattata alle variegate situazioni dimensionali dell'oggetto del rilievo. Va considerato che è preferibile un set numeroso, piuttosto che esiguo, infatti il programma ottiene risultati migliori con un maggior quantitativo di fotografie, l'appesantimento in termini di hardware per l'elaborazione è compensato dalla possibilità del software di ritrovare lo stesso dettaglio in più fotogrammi. Nelle impostazioni fotografiche è stata posta particolare attenzione al binomio profondità di campo - velocità dell'otturatore. Il problema delle foto mosse è stato affrontato in diversi articoli riguardanti le foto aeree eseguite tramite i droni, in quanto l'effetto scia crea un indeterminazione del pixel che ha ripercussioni negative nella fase di allineamento SFM. Per la fotogrammetria terrestre permangono le stesse considerazioni, ma a differenza delle foto aeree in cui la distanza della camera dall'oggetto è pressoché costante per ogni fotogramma, nelle foto terrestri vengono 
catturati oggetti anche a distanze intermedie. Anzi talvolta si esalta questo aspetto in quanto, come ormai assodato in letteratura per la fotogrammetria 3D, oggetti su piani diversi concorrono a migliorare l'unione, ne consegue l'attenzione per la profondità di campo, cioè la nitidezza accettabile sull'intero fotogramma. Diaframmi chiusi hanno come conseguenza tempi lunghi dell'otturatore e quindi il pericolo di foto mosse. Nel caso in esame si è quindi impostato un tempo dell'otturatore pari a $1 / 125$, con una sensibilità ISO 160 , il range $f$ dei diaframmi è variato tra 7,1 e 18 , con questi valori la profondità di campo con una focale di $28 \mathrm{~mm}$, ha assicurato nel caso di $f 7,1$ una messa a fuoco di oggetti compresi tra 2,69 m e infinito, nel caso di $f 18$ una messa a fuoco compresa tra 1,27 $\mathrm{m}$ e infinito. Casi particolari sono stati risolti singolarmente tenendo anche conto che l'ottica utilizzata è stabilizzata, e quindi è stato possibile allungare la velocità dell'otturatore. Poi in fase di elaborazione è stato eseguito un controllo selettivo scartando quelle foto che mosse o sfocate perdevano di nitidezza anche solo in alcune parti. Quindi si è calcolato per un campione di foto il Ground Sampling Distance medio nelle varie parti, tenendo conto dei parametri del sensore fotografico full frame $35,8 \times 23,9 \mathrm{~mm}$, il valore così ottenuto per le parti interne è stato di 2,3 $\mathrm{mm} /$ pixel, che per distanze dell'oggetto di circa $10 \mathrm{~m}$ significa un ricoprimento reale dell'immagine di 10,23 m di larghezza e 6,83 m di altezza. Per i muri esterni il GSD medio è di $2,7 \mathrm{~mm} /$ pixel con dimensione reali dell'immagine di 12,34 m x 9,1 m. Questi valori sono stati ritenuti validi per le finalità del rilievo, poiché hanno assicurato una buona definizione del dettaglio ad una scala di 1:50. Il software Zephyr prevede cinque fasi principali: l'allineamento fotografico, la creazione di una nuvola sparsa, la creazione di una nuvola densa, l'elaborazione di mesh, l'elaborazione di texture. Le fasi sono in sequenza e non è possibile ottenere separatamente migliori in una fase se nella precedente si sono avuti risultati inferiori. Le fasi più incisive, ai fini della generazione del modello $3 \mathrm{D}$, sono quelle della creazione della nuvola grezza e della nuvola densa. Le fasi di mesh e texture non migliorano il modello $3 \mathrm{D}$, ma con- sentono una migliore percezione fotorealistica. Considerata la notevole quantità di foto, sono state eseguite diverse elaborazioni parziali, scomponendo l'oggetto nelle sue parti, e lungo i suoi lati. Le prime elaborazioni sono state tentate in modalità automatica con i parametri di default per verificare la validità del set fotografico. Appurata l'elaborazione fotografica 3D dei singoli modelli coincidenti con i singoli parti del castello, si è proceduto all'elaborazione di un modello unico. Causa la notevole mole di dati, non è stato possibile settare i parametri al top a causa dei tempi di elaborazione. Quindi si è cercato un compromesso tra alcuni parametri sostanziali e i tempi di elaborazione, senza che ciò inficiasse la qualità dei risultati. Nella fase di creazione della nuvola sparsa, particolare attenzione è stata posta sui parametri di densità dei Keypoint e di profondità fase di matching. Il primo "progetto" complessivo, ha orientato un set di 719 foto su 819 , risultato ritenuto soddisfacente. Senza addentrarsi sulla scelta dei parametri, si vuole solo evidenziare che nella fase di estrazione della "nuvola densa" si è ottenuto un notevole miglioramento dei risultati variando il numero di $\mathrm{ca}$ mere vicine ai fini dell'elaborazione da 3 a 4.

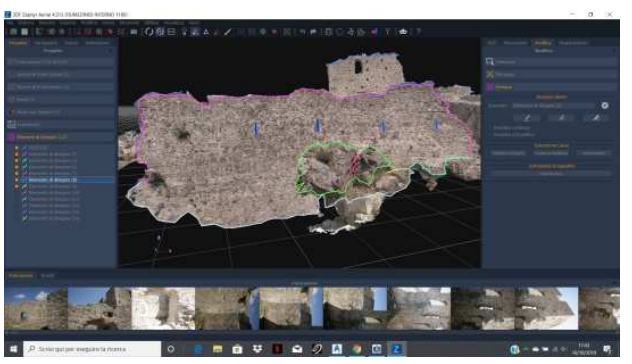

Fig. 5. Castello Belvedere, tracciamento grafico manuale in $3 \mathrm{D}$ del prospetto sud.

Il modello ottenuto tramite mesh e texture è stato scalato utilizzando la procedura che prevede le distanze di controllo, cioè l'utilizzo di alcune delle trilaterazioni rilevate con il Disto-D8 disposto orizzontalmente. Verificato il margine di tolleranza delle misure, si sono eseguite delle verifiche su altre parti dell'oggetto confrontando i risultati con le rimanenti trilaterazioni note. 


\section{La fase di restituzione}

La fase di restituzione grafica della pianta ha previsto l'integrazione di due diverse elaborazioni, la prima speditiva utilizzando il sistema di trilaterazioni di cui si è detto, ha consentito di ottenere un 2D vettoriale definendo il rilievo geometrico di massima. Per la seconda elaborazione effettuata a conclusione del processo 3D di mesh e texture, si è utilizzata l'ortofoto per descrive le aree di degrado dei ruderi presenti sulla sommità degli spalti. Per gli alzati è stata tentata l'esportazione del modello strutturato della mesh con texture in Autocad. I vari filtri presenti nelle procedure di esportazione e importazione hanno fatto perdere qualità al modello strutturato, con una scarsa definizione dei dettagli, inoltre la mole di dati del file non consente un'elaborazione agile in ambiente CAD. Sono state tentate delle elaborazioni anche con Rhinoceros, che fornisce diversi formati di interscambio di file, ma anche in questo caso faceva perdere qualità e risoluzione al modello strutturato. Infine si è reputato opportuno prendere in considerazione l'ambiente grafico presente in Zephyr, di cui è stato utilizzato l'editor grafico in continua evoluzione, che fornisce il tracciamento manuale. Per i prospetti è stato contornato il profilo di sommità del muro rivolto a sud-ovest, l'attacco a terra e le feritoie. L'obiettivo è stato quello di arrivare ad una restituzione 3D. Il notevole vantaggio è quello di seguire il contorno apparente degli oggetti del modello strutturato che presentano una notevole qualità visiva, nonché il tracciamento direttamente sul modello $3 \mathrm{D}$ continuo. Lo svantaggio è dato dalla limitatezza delle opzioni di un editor grafico primitivo carente di molti comandi essenziali. Tra queste carenze quelle più onerose sono riconducibili all'impossibilità di modifica della polilinea, e la mancanza degli snap elementari. Inoltre si è riscontrato che il tracciamento in 3D della polilinea, in prossimità di contorni discontinui, talvolta aggancia punti appartenenti ad oggetti disposti su piani differenti, di solito retrostanti (Fig. 7). Di questo scarto è possibile accorgersi solo a completamento della restituzione, orbitando la vista, la semplice correzione non è fattibile, come già detto, non essendo possibile modificare la polilinea, la soluzione consiste nel rieseguire tutto il tracciamento. I limiti anzidetti sono stati segnalati alla 3Dflow, che oltre ad adoperarsi rapidamente per tali miglioramenti, ha chiesto all'autore dell' articolo di intervenire come beta tester dell'editor grafico. Attualmente per il castello Belvedere si sta ultimando la costruzione del modello 3D, completando le zone non definite dalle immagini fotografiche. Tra i prossimi obiettivi è prevista l'analisi grafica delle piante e dei prospetti.

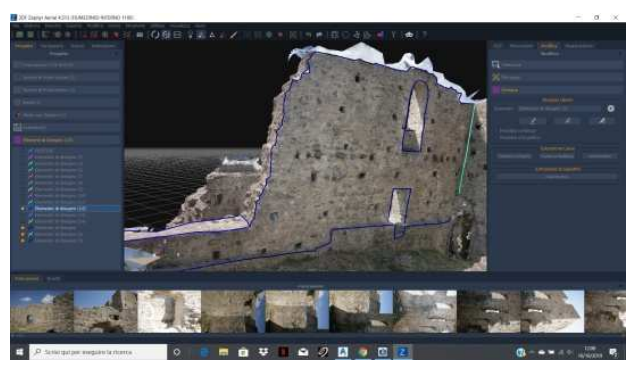

Fig. 6. Castello Belvedere, parte interna: tracciamento grafico manuale in $3 \mathrm{D}$ del nucleo a due elevazioni.

\section{Conclusioni}

Il concetto di sostenibilità, non certo recente, rimane pur sempre attuale. La gestione e programmazione degli interventi e quindi le priorità del patrimonio culturale sono di difficile applicazione, si ritiene tuttavia che bisogna fare una distinzione tra interventi di restauro e interventi volti all'analisi conoscitiva. Il divario tra i due in termini di costi e tempi è notevole, ma se è vero che non si può intervenire in termini economici sull'intero patrimonio del nostro paese, è pur vero che si ha il dovere di prevedere e approntare studi e analisi conoscitive di questo patrimonio, monitorando e schedando le emergenze architettoniche e archeologiche attraverso operazioni di rilievo. I manufatti più deboli del territorio sono quelli dimenticati dalla collettività più ampia, e conosciuti solo da quella locale, che ne conserva memoria e tradizione, come nel caso in questione. Basti pensare che di alcune emergenze archeologiche già nei secoli passati si erano perse le tracce e sono state poi portate alla luce solo grazie alle fonti documentarie. Vale per tutti il caso del Teatro Greco di Palazzolo Acreide, del sito di Morgantina nonché della Villa Romana del Casale. Ma l'elenco solo in Sicilia è molto più ampio. Nel caso in esame si sono mostrati i 
risultati ottenuti con un sistema a basso costo e alla portata di molti. Si ritiene che per oggetti del patrimonio culturale appartenenti a questa tipologia, i sistemi di rilievo di fotogrammetria SFM possano essere ritenuti efficaci e speditivi, va però detto che sebbene utilizzati da tempo da archeologi e professionisti, sono stati sperimentati prevalentemente per oggetti geometricamente regolari, ma ancora non sono arrivati ad un punto accettabile per oggetti irregolari. La loro continua evoluzione richiede continui test operativi per le diverse innovazioni, non ultima la parte dell'editor grafico che è stata implementata solo successivamente ai moduli dense matching. La rapida e continua evoluzione del software è assodata, basti pensare che nel corso della stesura del presente articolo sono state rilasciate diversi aggiornamenti. Pur tuttavia va riconosciuto che consentono di affrontare il rilievo con tempi e costi fino a qualche tempo fa impensabili.

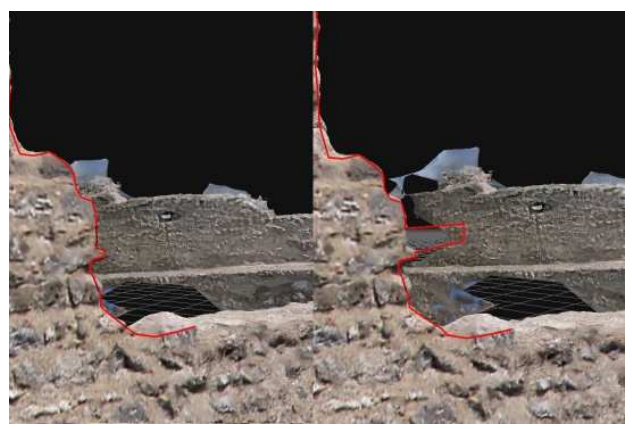

Fig. 7. Fase di restituzione. Situazione critica di un tracciamento: la polilinea dello stesso profilo da due viste differenti.

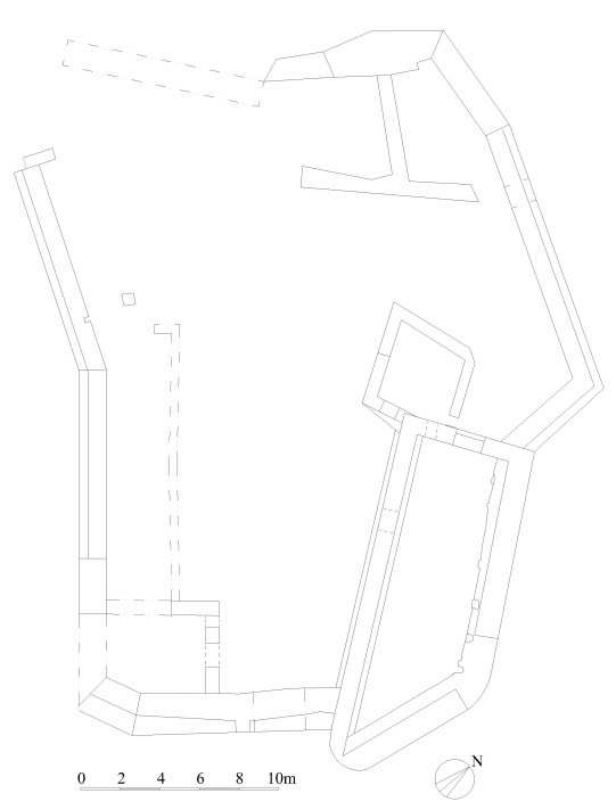

Fig. 8. Castello Belvedere, la pianta.

\section{Note \\ ${ }^{1}$ Gregorio, 2013, p. 224. L'autore riporta che una recente pubblicazione ricorda una Torre Rossa nel territorio di Fiumedinisi.}

\section{Bibliography}

Amari, M. (1889). Biblioteca Araba - Sicula, First Ed., Torino.

Amari, M. (1935). Storia dei Musulmani in Sicilia, Nabu Press Ed., Catania.

Amoruso, G.; Cochelli, P.; Riavis, V. (2018). "Il Castello di Gorizia, analisi geometrica e rilievo con metodologie avanzate", in Marotta, A.; Spallone, R., eds., FORTMED 2018. Defensive Architecture of the mediterranean, Politecnico di Torino, Torino, vol. IX, p. 933-940.

Arena, M.; Fatta, F. (2018). "Turris ad noctorum navigantibus lumen”, in Marotta, A.; Spallone, R. eds., FORTMED 2018. Defensive Architecture of the mediterranean, Politecnico di Torino, Torino, vol. IX, pp. 941-948

Bacci, G.M. (1983). "Fiumedinisi 1978-1979”, Beni Culturali e Ambientali - Sicilia, III, 1-2-3-4, Palermo.

Bertocci, S.; Lumini A. (2018). "Rilievo digitale dell' area archeologica costiera della Rocca di San Silvestro", in Marotta, A.; Spallone, R., eds., FORTMED 2018. Defensive Architecture of the mediterranean, Politecnico di Torino, Torino, vol. IX, pp. 957-964. 
Bigongiari, M. (2018). "Il castello di Brolo, rilievo digitale integrato per la conservazione del Patrimonio", in Marotta, A.; Spallone, R., eds., FORTMED 2018. Defensive Architecture of the mediterranean, Politecnico di Torino, Torino, vol. VIII, pp. 441-448.

Gregorio, C. (2013). Storie e segreti di Fiuedinisi, Nuova Prhomos Editore, Castello.

Maurici, F. (1990). "Le fortezze della Sicilia musulmana”, Beni Culturali e Ambientali, IX-X, 1-2, pp. 11-41.

Pettineo, A. (2006). Testimonianze della provincia Bizantina in Val Demone, Quartarone, C. ed., Sicilia Romana e Bizantina, Palermo.

Riccobono, F. (1981). Monte Scuderi, storia e leggenda, Messina Ed.

Vallone, G. (2005). I Castelli della Sicilia, Newton Compton Editori, Roma. 